\title{
FPGA BASED ADAPTIVE NEURO FUZZY INFERENCE CONTROLLER FOR FULL VEHICLE NONLINEAR ACTIVE SUSPENSION SYSTEMS
}

\author{
Ammar A. Aldair ${ }^{1}$ and Weiji Wang ${ }^{2}$ \\ School of Engineering and Design, University of Sussex, Falmer, East Sussex Brighton, \\ BN1 9QT, UK \\ 1 aa386esussex.ac.uk $\quad$ w.j.wang@sussex.ac.uk
}

\begin{abstract}
A Field Programmable Gate Array (FPGA) is proposed to build an Adaptive Neuro Fuzzy Inference System (ANFIS) for controlling a full vehicle nonlinear active suspension system. A Very High speed integrated circuit Hardware Description Language (VHDL) has been used to implement the proposed controller. An optimal Fraction Order $\mathrm{PI}^{\lambda} D^{\mu}$ (FOPID) controller is designed for a full vehicle nonlinear active suspension system. Evolutionary Algorithm (EA) has been applied to modify the five parameters of the FOPID controller (i.e. proportional constant $K_{p}$, integral constant $K_{i}$, derivative constant $K_{d}$, integral order $\lambda$ and derivative order $\mu$ ). The data obtained from the FOPID controller are used as a reference to design the ANFIS model as a controller for the controlled system. A hybrid approach is introduced to train the ANFIS. A Matlab Program has been used to design and simulate the proposed controller. The ANFIS control parameters obtained from the Matlab program are used to write the VHDL codes. Hardware implementation of the FPGA is dependent on the configuration file obtained from the VHDL program. The experimental results have proved the efficiency and robustness of the hardware implementation for the proposed controller. It provides a novel technique to be used to design NF controller for full vehicle nonlinear active suspension systems with hydraulic actuators.
\end{abstract}

\section{KEYWORDS}

Full vehicle, Nonlinear Active Suspension System, Neuro-fuzzy Control, FPGA, Hardware Implementation.

\section{INTRODUCTION}

The conventional controller like the PID controller requires an exact mathematical model of the controlled system to meet as much control objectives as possible. If it is difficult to establish the mathematical model for a system, the fuzzy logic controller is a good option to achieve a robust controller. Fuzzy logic systems, which can reason with imprecise information, are good at explaining their decisions but they cannot automatically acquire the rules used to make those decisions. On the other hand, artificial neural networks are good at recognizing patterns, and have ability to train the parameters of a control system, but they are not good at explaining how they reach their decisions. These limitations in both systems have brought about driving force behind the creation of intelligent hybrid systems (like Neuro-fuzzy system) where the two techniques are combined in a manner that the limitations of the individual techniques have been overcome. The neuro-adaptive learning techniques provide a method for the fuzzy modelling procedure to acquire information about a data set. This technique gives the fuzzy logic capability to compute the membership function parameters that effectively allow the associated fuzzy inference system to track the given input and output data. In order to process a fuzzy rule 
by neural networks, it is necessary to modify the standard neural network structure accordingly. In the last two decades, many researchers focused on the development of a hardware implementation for both fuzzy logic controller and neural controller. [1], [2] and [3] used an analogue circuit to implement each part of fuzzy system (including: Fuzzification, Fuzzy Inference and Defuzzification). The structure of fuzzy system is complex, so that the analogue circuit has to be very complicated to implement the logic system. Therefore, many researchers proposed digital rather than analogue circuits to implement the fuzzy logic system. Microprocessors or microcontrollers are popular in being used to implement fuzzy logic system or neural network. Microprocessor based controllers are economical and flexible, but often face difficulties in dealing with control systems. Therefore, higher density programmable logic devices such as Programmable Logic Device (PLD) and Field Programmable Gate Array (FPGA) have been developed to overcome the problems of microprocessors. The FPGA is suitable for fast implementation and hardware verification. The control systems based on it are flexible and can be reprogrammed with unlimited number of times. Many papers have reported this technology to design Fuzzy Logic Controller (FLC) and Neural Controller (NC) for different applications. Reference [4] developed software for synthesizing fuzzy controllers into Boolean equations. Also, a hardware implementation of a fuzzy controller on the FPGA has been described. The developed software together with the FPGA development system provides a complete automation design tool for fuzzy controllers. Reference [5] presented an analysis and performance evaluation of the proportional-derivative (PD) fuzzy logic controller designs using Matlab and field programmable gate array. An embedded run-time reconfigurable architecture for the design of a fuzzy logic PID controller is proposed in [6], [7] and [8]. Reference [9] presented and implemented a fuzzy controller on an FPGA using VHDL for a motor unit. A Fuzzy controller has been implemented on the FPGA board to control a shunt motor used for controlling the speed of electrical vehicle [10]. In Reference [11] simulation and implementation of a fuzzy logic controller for a diesel driven stand-alone synchronous generator have been designed. The controller was developed using VHDL and implemented in FPGA. Reference [12] depicted a fuzzy logic model style based on two strategies: behavioral modelling using VHDL and structural VHDL based on specific architecture of a fuzzy processor. Reference [13] described the hardware implementation of fuzzy systems, a neural networks and fuzzy neural networks using Xilinx FPGA.

The neuro-adaptive learning techniques provide a method for a fuzzy modelling procedure to learn information about data sets. This technique gives the fuzzy logic capability to compute the membership function parameters that best allow the associated fuzzy inference system to track the given input and output data.

In this paper, an Adaptive Neuro-Fuzzy Inference System (ANFIS) based intelligent control for full vehicle nonlinear active suspension system will be designed. The FOPID sub-controller will be used to control the master controlled system. An Evolutionary Algorithm is proposed to modify the control parameters of FOPID. The data obtained from the FOPID controller will be used as a reference to train the parameters of the Neuro-fuzzy controller. The Simulink tool boxes in Matlab will be used to simulate the controlled system (full vehicle nonlinear active suspension system) with the proposed controller. The VHDL has been used to describe the implementation of Neuro-fuzzy controller. In the FPGA based design, Xilinx ISE Project Navigator Version 10.1 is used to obtain the compilation and timing test results as well as the synthesized design. For the simulation results, ModelSim XE III 6.4b simulation program will be used with the FPGA-based design. In order to compare the responses of the FPGA design with the Simulink design, an M-file (Matlab-file) will be used to plot the data collected from the ModelSim program and the other data collected form the Simulink design. These comparisons will show that the responses of the FPGA design are similar to the responses of the Simulink design. 


\section{Mathematical Model of The Controlled System}

The main purposes of active suspension controller are to increase both riding comfort and handling quality. The riding comfort can be measured by evaluating the acceleration and displacement of sprung mass. The handling quality can be achieved by controlling the rotational motions of the vehicle body such as rolling and pitch movements during cornering and braking. Figure 1 illustrates the full vehicle nonlinear active suspension with hydraulic actuators. In this model the tyres are modeled as linear spring in parallel with linear viscous dampers. However, the suspension part is modeled as a nonlinear spring in parallel with a nonlinear damper and a nonlinear hydraulic actuator. The nonlinear frictional forces due to rubbing of piston seals with the cylinders wall inside the actuators are taken into account to calculate the real supply forces generated by the hydraulic actuator

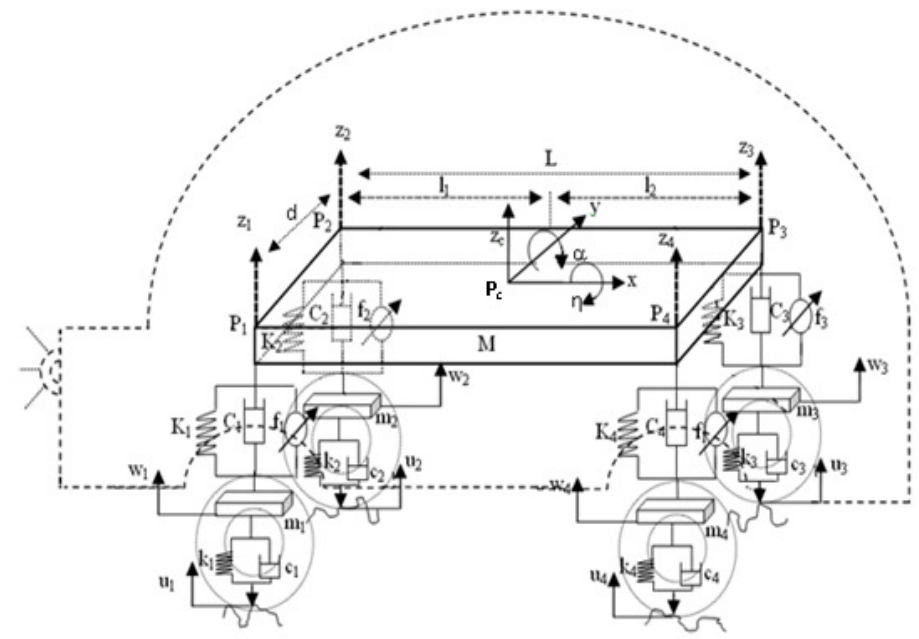

Figure 1. Full Vehicle Nonlinear Active Suspension

The following equations of motion are derived for the model using Newton laws of motion:

\section{Vertical motion}

\section{According to}

$M \ddot{z}_{c}=-\sum_{i=1}^{4} F_{K i}-\sum_{i=1}^{4} F_{C i}+\sum_{i=1}^{4} F_{P i}$

where $F_{K i}$ and $F_{C i}$ are the nonlinear suspension spring force and nonlinear suspension damping force, respectively, which can be written as [14]

$$
\begin{gathered}
F_{K i}=K_{i}\left(z_{i}-w_{i}\right)+\xi K_{i}\left(z_{i}-w_{i}\right)^{3} \\
F_{c i}=C_{i}\left(\dot{z}_{i}-\dot{w}_{i}\right)+\xi C_{i}\left(\dot{z}_{i}-\dot{w}_{i}\right)^{2} \operatorname{sgn}\left(\dot{z}_{i}-\dot{w}_{i}\right)
\end{gathered}
$$

The force generated by hydraulic actuator can be written as

$$
F_{P i}=F_{A i}-F_{f i}
$$

where $F_{A i}$ is the nonlinear hydraulic force provided by the $i^{\text {th }}$ actuator and $F_{f i}$ the nonlinear frictional force due to rubbing of piston seals with the cylinder wall inside the $i^{\text {th }}$ actuator. The 
relation between the spool valve velocity, $\dot{x}_{v i}$, and the output force of this actuator, $F_{A i}=A_{p} P_{L i}$, possess a nonlinear dynamic behaviour [15].

$$
\dot{F}_{A i}=A_{p} \alpha\left[C_{d} \omega x_{v i} \sqrt{\frac{P_{s i}-s g n\left(x_{v i}\right) P_{L i}}{\rho}}-C_{t m} P_{L i}-A_{p}\left(\dot{z}_{i}-\dot{w}_{i}\right)\right]
$$

The frictional force is modeled with a smooth approximation of Signum function:

$$
F_{f i}=\left\{\begin{array}{l}
\kappa \operatorname{sgn}\left(\dot{z}_{i}-\dot{w}_{i}\right) \text { if }\left|\dot{z}_{i}-\dot{w}_{i}\right|>0.01 \\
\kappa \sin \left(\frac{\dot{z}_{i}-\dot{w}_{i}}{0.01} \frac{\pi}{2}\right) \text { if }\left|\dot{z}_{i}-\dot{w}_{i}\right|<0.01
\end{array}\right.
$$

\section{Pitching motion}

$$
\begin{gathered}
J_{x} \ddot{\alpha}=\left(F_{K 1}-F_{K 2}-F_{K 3}+F_{K 4}\right) \frac{b}{2}+ \\
\left(F_{C 1}-F_{C 2}-F_{C 3}+F_{C 4}\right) \frac{b}{2}+ \\
\left(F_{P 4}-F_{P 1}+F_{P 3}-F_{P 2}\right) \frac{b}{2}+T_{x}
\end{gathered}
$$

where $\mathrm{b}$ is the distance between the front wheels (or rear wheels).

\section{Rolling motion}

$$
\begin{aligned}
J_{y} \ddot{\eta}= & \left(F_{K 3}+F_{K 4}\right) l_{2}-\left(F_{K 1}+F_{K 2}\right) l_{1}+ \\
& \left(F_{C 3}+F_{C 4}\right) l_{2}-\left(F_{C 1}+F_{C 2}\right) l_{1}+ \\
& \left(F_{P 1}+F_{P 2}\right) l_{1}+\left(F_{P 3}+F_{P 4}\right) l_{2}+T_{y}
\end{aligned}
$$

where $l_{1}$ is the distance between the centre of front wheel axle and centre of gravity of the vehicle. $l_{2}$ is the distance between the centre of gravity of the vehicle and the centre of rare wheel axle.

The motion of the $i^{\text {th }}$ unsprung mass is governed by the following equation:

$m_{i} \ddot{w}_{i}=-k_{i}\left(w_{i}-u_{i}\right)-c_{i}\left(\dot{w}_{i}-\dot{u}_{i}\right)+F_{K i}+F_{C i}-F_{P i}$

The experimental values for the above parameters are shown in Table 1.

\section{Fractional-Order PI ${ }^{\lambda} D^{\mu}$ CONTROller}

A fractional order differential equation is used to describe the fractional order $\mathrm{PI}^{\lambda} \mathrm{D}^{\mu}$ controller. In PID controller case, three parameters $K_{p}, K_{d}$ and $K_{i}$ should be tuned to optimize the controller. One of the possibilities to improve PID controllers is to use fractional-order controllers with a real order of derivative and integral. The differential equation of fractional order controller can be described by

$$
u(t)=K_{p} e(t)+K_{i} D_{t}^{-\lambda} e(t)+K_{d} D_{t}^{\mu} e(t)
$$


where $e(t)$ is the error between a measured process output variable and a desired set point and $u(t)$ is the control output. Eq. (7) shows that the FOPID controller needs to tune five parameters: $K_{p}$, $K_{i}, K_{d}, \lambda$ and $\mu$. Therefore, the integral order and derivative order add more flexibility to design an FOPID controller.

The continuous transfer function of the FOPID is given by

$$
G_{c}(s)=\frac{U(s)}{E(s)}=K_{p}+\frac{K_{i}}{s^{\lambda}}+K_{d} s^{\mu}
$$

The big challenge is how the optimal parameters of the FOPID controller can be chosen for designing an accurate controller. An Evolutionary Algorithm has been used to serve this purpose.

Table 1.Vehicle Suspension Parameters

\begin{tabular}{|c|c|c|c|}
\hline Notation & Description & Values & Units \\
\hline$K_{1}, K_{2}$ & $\begin{array}{l}\text { Front-left and Front-right } \\
\text { suspension stuffiness, } \\
\text { respectively. }\end{array}$ & 19960 & $\mathrm{~N} / \mathrm{m}$ \\
\hline$K_{3}, K_{4}$ & $\begin{array}{l}\text { Rear-right and rear-left } \\
\text { suspension stuffiness, } \\
\text { respectively. }\end{array}$ & 17500 & $\mathrm{~N} / \mathrm{m}$ \\
\hline$k_{1}-k_{4}$ & $\begin{array}{l}\text { Front-left, Front-right, rear- } \\
\text { right and rear-left tire } \\
\text { stuffiness respectively. }\end{array}$ & 175500 & $\mathrm{~N} / \mathrm{m}$ \\
\hline$C_{1}, C_{2}$ & $\begin{array}{l}\text { Front-left and Front-right } \\
\text { suspension damping, } \\
\text { respectively. }\end{array}$ & 1290 & N.sec/m \\
\hline$C_{3}, C_{4}$ & $\begin{array}{l}\text { Rear-right and rear-left } \\
\text { suspension stuffiness, } \\
\text { respectively. }\end{array}$ & 1620 & N.sec/m \\
\hline$c_{1}-c_{4}$ & $\begin{array}{l}\text { Front-left, Front-right, rear- } \\
\text { right and rear-left tire } \\
\text { damping, respectively. }\end{array}$ & 14.6 & N.sec/m \\
\hline$M$ & Sprung mass. & 1460 & $\mathrm{~kg}$ \\
\hline$m_{1}, m_{2}$ & $\begin{array}{l}\text { Front-left, Front-right tire } \\
\text { mass, respectively. }\end{array}$ & 40 & $\mathrm{~kg}$ \\
\hline$m_{3}, m_{4}$ & $\begin{array}{l}\text { Rear-right and rear-left tire } \\
\text { mass, respectively. }\end{array}$ & 35.5 & $\mathrm{~kg}$ \\
\hline$J_{x}$ & $\begin{array}{l}\text { Moment of inertia } \mathrm{X}^{-} \\
\text {direction. }\end{array}$ & 460 & $\mathrm{~kg} \cdot \mathrm{m}^{2}$ \\
\hline$J_{y}$ & $\begin{array}{l}\text { Moment of inertia y- } \\
\text { direction. }\end{array}$ & 2460 & $\mathrm{~kg} \cdot \mathrm{m}^{2}$ \\
\hline$l_{1}$ & $\begin{array}{l}\text { Distance between the center } \\
\text { of gravity of vehicle body } \\
\text { and front axle. }\end{array}$ & 1.011 & $\mathrm{~m}$ \\
\hline$l_{2}$ & $\begin{array}{l}\text { Distance between the center } \\
\text { of gravity of vehicle body } \\
\text { and rear axle. }\end{array}$ & 1.803 & $\mathrm{~m}$ \\
\hline
\end{tabular}


International Journal of Artificial Intelligence \& Applications (IJAIA), Vol.1, No.4, October 2010

\begin{tabular}{|c|l|c|c|}
\hline$b$ & Width of vehicle body & 1.51 & $\mathrm{~m}$ \\
\hline$\zeta$ & Empirical parameter & 0.1 & - \\
\hline$\alpha, \beta, \gamma$ & Actuator parameters & $\begin{array}{c}4.515^{*} 10^{13} \\
, 1, \\
1.545^{*} 10^{9}\end{array}$ & - \\
\hline$A_{P}$ & Cross section area of piston & $3.35^{*} 10^{-4}$ & $\mathrm{~m}^{2}$ \\
\hline$P_{S}$ & Supply pressure & 10342500 & $\mathrm{~Pa}$ \\
\hline$\tau$ & Time constant & $1 / 30$ & $\mathrm{sec}$ \\
\hline$C_{\mathrm{d}}$ & Discharge coefficient & 0.7 & - \\
\hline$\rho$ & Fluid density & 970 & $\mathrm{~kg} / \mathrm{m}^{3}$ \\
\hline$\omega$ & Area gradient & $1.436 \mathrm{e}-2$ & $\mathrm{~m}^{2}$ \\
\hline
\end{tabular}

\section{The Structure AND Its training of Adaptive Neuro Fuzzy INFERENCE SYSTEM (ANFIS)}

The ANFIS is one of the methods to organize the fuzzy inference system with given input-output data pairs. The ANFIS is a combination of a fuzzy logic controller and a neural network, which enables the controller self-tuning and adaptive. If these two intelligent techniques are combined, it will achieve good reasoning in quality and quantity. This approach enables the fuzzy logic capability to adapt the membership function parameters that best allow the associated fuzzy inference system to track the given input and output data. The data obtained from the FOPID controller will be use to modify the parameters of the ANFIS model. In order to process a fuzzy rule by neural networks, it is necessary to modify the standard neural network structure accordingly. Figure 2 depicts the structure of Neuro-fuzzy inference system (the type is called Takagi-Sugeno-Kang [18]). For simplicity, the following assumptions will be made: (a) the model has two inputs $x$ and $y$ and one output $z$,(b) it has just two rules ( $R 1$ and $R 2)$.

R1: If $x$ is $A_{1}$ and $y$ is $B_{1}$ then $f_{1}=p_{1} x+q_{1} y+r_{1}$

R2: If $x$ is $A_{2}$ andy is $B_{1}$ then $f_{2}=p_{2} x+q_{1} y+r_{2}$

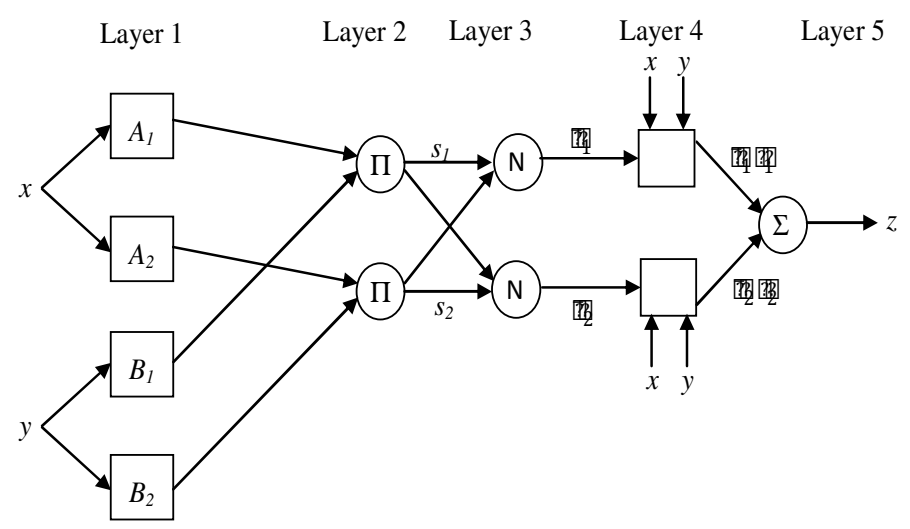

Figure 2. ANFS model structure

In Figure 2, the square nodes (adaptive nodes) have adaptable parameters while the circle nodes (fixed nodes) have non-adaptable parameters. The function of each layer is described below: 
Layer 1: Every node $I$ in this layer is a square node with a node function

$O_{i}^{1}=\mu_{A_{i}}(w) \quad i=1,2$

where $w$ is the input to the node $i$ ( $x$ or $y$ ) and $A_{i}$ is the linguistic label associated with this node. $\mu_{A_{i}}(w)$ has been chosen as bell shape membership function:

$\mu_{A_{i}}(w)=\frac{1}{1+\left[\left(\frac{w-c_{i}}{a_{i}}\right)^{2}\right]^{b_{i}}}$

where $\left\{a_{i}, b_{i}, c_{i}\right\}$ are the parameters of membership function (they are called premise parameters) which will be modified in the training phase.

Layer 2: Every node in this layer is a circle node (has non-adaptable parameters) labeled $\Pi$ which multiplies the incoming signals. The output of each node in this layer can be written as:

$O_{i}^{2}=s_{i}=\mu_{A_{i}}(x) \mathrm{X} \mu_{B_{i}}(y) \quad i=1,2$

Layer 3: Every node in this layer is a circle node labeled $N$. the output of $i^{\text {th }}$ node is the normalized of the $i^{\text {th }}$ rule's firing strength. The output of any node in this layer can be given as

$O_{i}^{3}=\bar{s}_{i}=\frac{s_{i}}{s_{1}+s_{2}}$

Layer 4: Every node in this layer is square node with a linear function

$f_{i}=p_{i} x+q_{i} y+r_{i}$

where $\left\{p_{i}, q_{i}, r_{i}\right\}$ is the set parameters called consequent parameters of linear equation which will be modified in the training phase. The output of any nodes in this layer can be written as:

$O_{i}^{4}=\bar{s}_{i} f_{i}=\bar{s}_{i}\left(p_{i} x+q_{i} y+r_{i}\right)$

Layer 5: the node in this layer is circle node labeled $\Sigma$ that computes the overall output as the summation of all incoming signals

$z=O_{i}^{5}=\sum_{i} \bar{s}_{i} f_{i}=\frac{\sum_{i} s_{i} f_{i}}{\sum_{i} s_{i}}$

The adaptable parameters of the ANFIS $\left\{a_{i}, b_{i}, c_{i}, p_{i}, q_{i}, r_{i}\right\}$ should be modified to minimize the following performance function:

$E=\sum_{p=1}^{P} E_{p}$

where $P$ is the total number of training data set and $E_{p}$ the error signal between the desired output of $p^{\text {th }}$ data and the actual output of the ANFIS model of $p^{\text {th }}$ data. $E_{p}$ can be given by

$E_{p}=T_{p}-z_{p}$ 
where $T_{p}$ is the $p^{\text {th }}$ desired output and $z_{p}$ is the $p^{\text {th }}$ actual output in the ANFIS model.

To modify the parameters of the ANFIS model, the steepest descent method as in neural network can be applied to modify the premise parameters $\left\{a_{i}, b_{i}, c_{i}\right\}$ and least square estimate can be applied to adapt the consequent parameters $\left\{p_{i}, q_{i}, r_{i}\right\}$ [19].

\section{HARDWARE DESCRIPTION OF ANFIS}

The VHDL codes have been developed to describe the ANFIS model controller. The optimal values of the premise parameters and the consequent parameters have been used to write the VHDL codes. Xilinx ISE 10.1 has been used as programming environment to write these codes. To generate a configuration file used to program the FPGA board, the flowing steps should be followed:

i. Create the design project and VHDL codes,

ii. Create a testbench and perform RTL simulation,

iii. Add a constraint file then synthesize and implement the codes.

After downloading the configuration file on to the FPGA chip (XILINX Spartan XC3S700AN) the FPGA board will be ready to use as ANFIS controller for the controlled system. The Spartan 3E starter Kit board highlights the unique features of the Spartan_3E FPGA family and provides a convenient development board for embedded processing applications. The Hirose 100-pin FX2 Edge connector (J3) will be used as input and output port to receive the digital input data (error and error rate) from the A/D converter (error signal is the difference between the output of the controlled system and desired output) and send the digital control signal to the D/A converter (analogue control signal will be used to force the output of the controlled system to follow the desired output).

\section{Simulation AND ReSults}

The active suspension is presented in order to reduce the discomfort arising from road roughness and to improve the handling quality. This necessitates a very fast and accurate controller to meet as much control objectives as possible. Furthermore, the controlled system has very complex nonlinear model as shown in Section 2. Therefore, an intelligent Neuro fuzzy controller has been proposed to meet the control objectives. An Evolutionary Algorithm has been applied to modify the five parameters (proportional constant $K_{p}$, integral constant $K_{i}$, derivative constant $K_{d}$, integral order $\lambda$ and derivative order $\mu$ ) of each FOPID controller. Figures 3, 4, 5, 6 and 7 show the changing of the control parameters during the optimization process. After 225 optimization iteration steps, the optimal values of the FOPID controller parameters can be obtained as shown in Table 2. During optimization phase, it is assumed that the inputs to the full vehicle model are just the road uneven excitation and control forces. The white noise input is supplied as road profile. 
International Journal of Artificial Intelligence \& Applications (IJAIA), Vol.1, No.4, October 2010

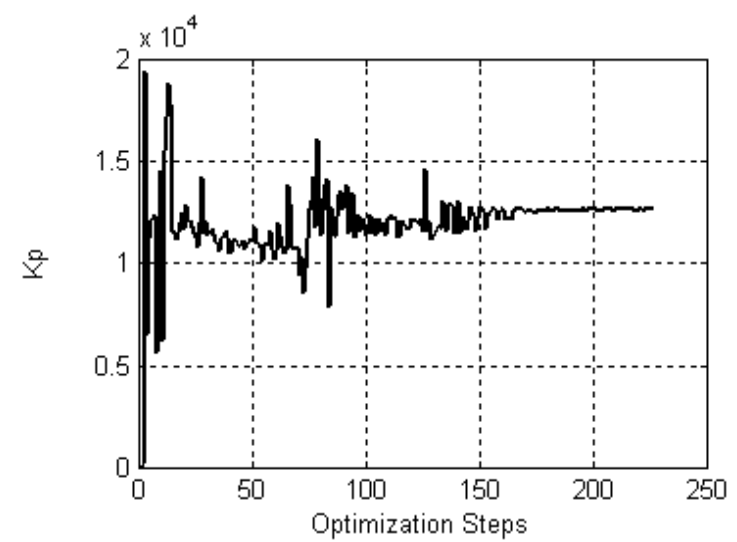

Figure 3. Changing value of $K_{p}$ during optimization steps

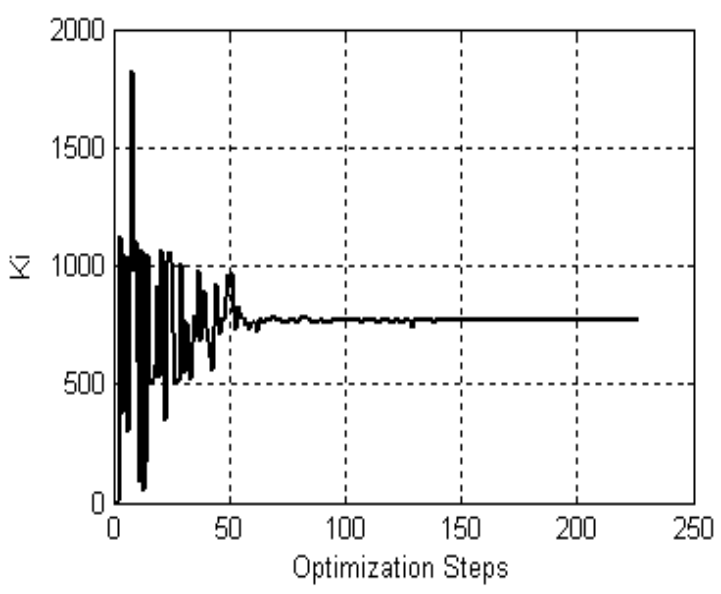

Figure 5. Changing value of $\mathrm{K}_{\mathrm{i}}$ during optimization steps

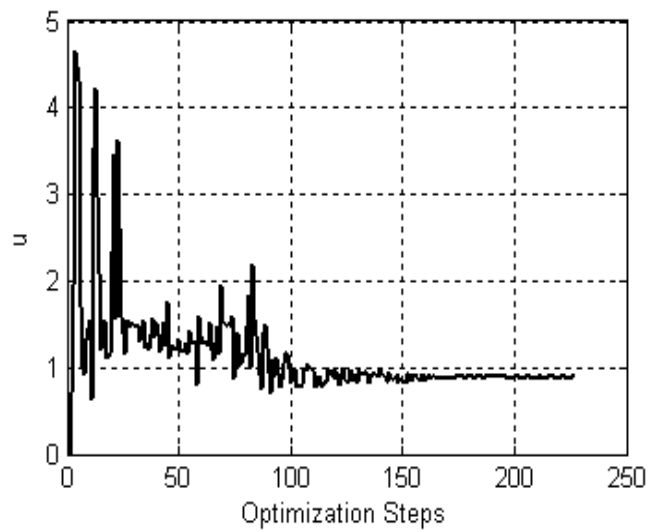

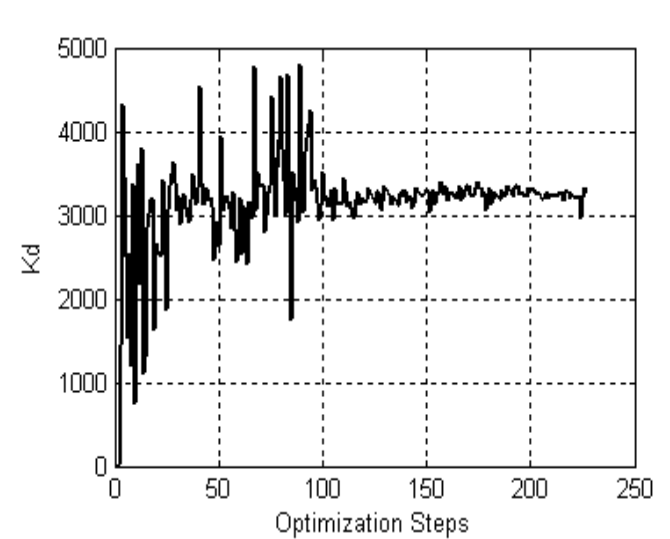

Figure 4. Changing value of $\mathrm{K}_{\mathrm{d}}$ during optimization steps

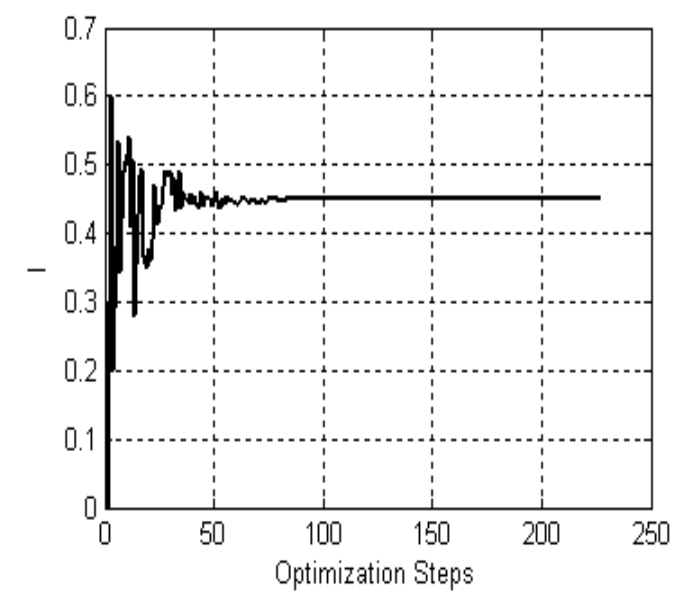

Figure 6 . Changing value of $\lambda$ during optimization steps

Table 2. Initial and optimal values of FOPID controller

\begin{tabular}{|c|c|c|}
\hline Parameter & Initial value & $\begin{array}{c}\text { Optimal } \\
\text { value }\end{array}$ \\
\hline $\mathbf{K}_{\mathbf{P}}$ & $\mathbf{1 0 0}$ & $\mathbf{1 2 6 7 8 . 2 6}$ \\
\hline $\mathbf{K}_{\mathbf{d}}$ & $\mathbf{2 0}$ & $\mathbf{3 2 5 3 . 9 2}$ \\
\hline $\mathbf{K}_{\mathbf{i}}$ & $\mathbf{1}$ & $\mathbf{7 6 8 . 1}$ \\
\hline$\lambda$ & $\mathbf{0 . 3}$ & $\mathbf{0 . 4 5}$ \\
\hline$\mu$ & $\mathbf{0 . 7}$ & $\mathbf{0 . 8 8 6}$ \\
\hline
\end{tabular}

Figure 7. Changing value of $\mu$ during optimization steps 
The hybrid learning algorithm has been used to modify the ANFIS (premise and consequent) parameters to track the input and output data obtained from FOPID controller. Four NF controllers have been designed (one for each suspension).

To establish the effectiveness of the proposed controller the robustness should be examined. Four types of disturbances are applied in turn to check the robustness of the NF controller as described below.

\section{- Square input signal with varying amplitude applied as road input profile}

The square input signal has been applied as road input. The amplitude of this signal has been changed from $0.01 \mathrm{~m}$ to $0.1 \mathrm{~m}$. At each value the cost function (as described in Equation 17) has been calculated:

$$
\phi=0.5 \sum_{\varepsilon=1}^{4} \mathrm{z}_{\varepsilon}^{2}
$$

Figure 8 shows the time response of the cost function as function of amplitude of square signal input.

\section{- Sine wave input signal with varying amplitude applied as road input profile}

The different amplitude of sine wave input from $0.01 \mathrm{~m}$ to $0.1 \mathrm{~m}$ has been applied as road profile input. The time response of the cost function for the full vehicle without controller, the result of the FOPID controller and NF controller are shown together in Figure 9 (where the scale of the $y$-axis is the log scale).

\section{- Bending inertia Torque (Tx) applied}

The value of bending torque (from $1000 \mathrm{Nm}$ to $9000 \mathrm{Nm}$ ) in addition to random signal as road profile has been applied. The cost function response is plotted as function of $T_{x}$ in Figure 10.

\section{- Breaking inertia Torque (Ty) applied}

The value of breaking torque (from $1000 \mathrm{Nm}$ to $9000 \mathrm{Nm}$ ) in addition to random signal as road profile has been applied. The cost function response is plotted as function of $T_{y}$ in Figure 11.

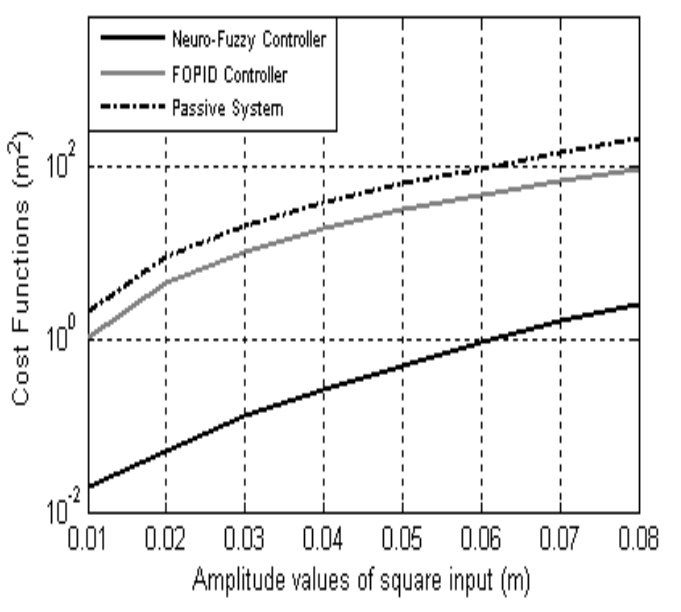

Figure 8. Time response of the cost functions against the different amplitude of square input

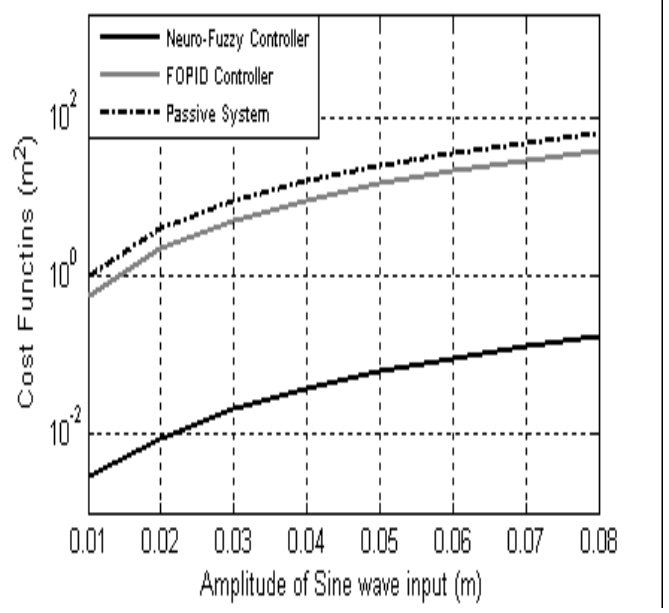

Figure 9. Time response of the cost functions against the different amplitude of sine wave input. 


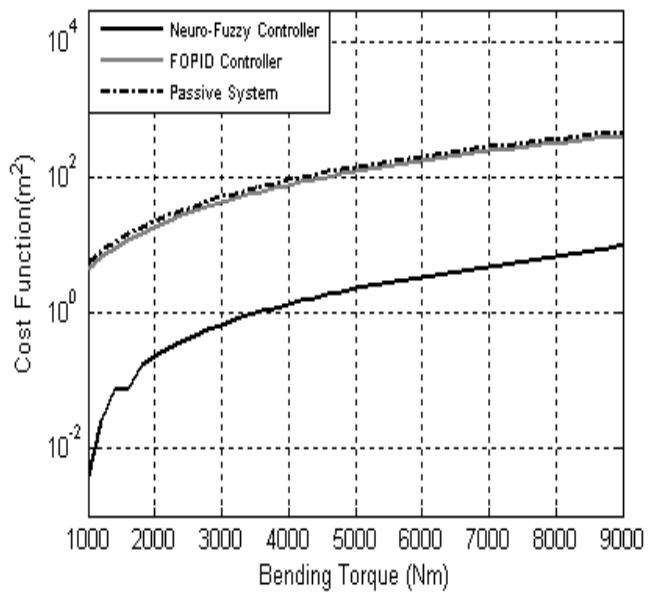

Figure 10. Time response of the cost functions against bending torque $\left(\mathrm{T}_{\mathrm{x}}\right)$

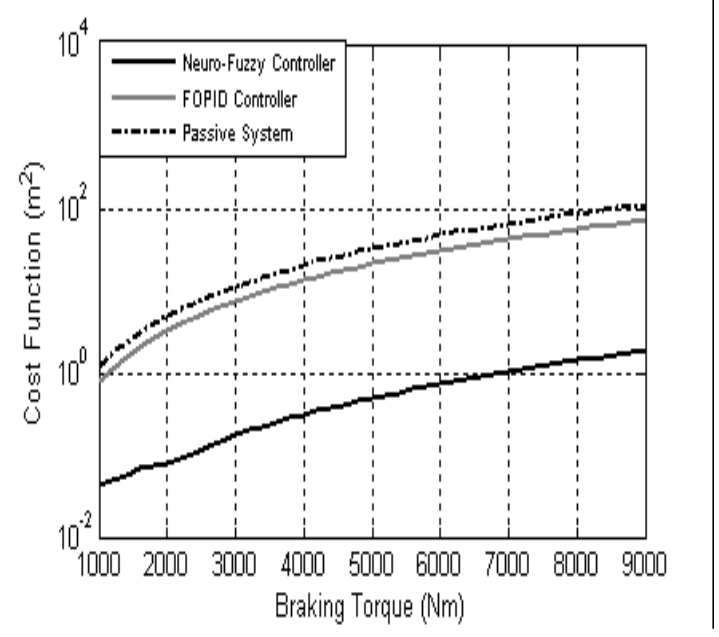

Figure 11. Time response of the cost functions against braking torque $\left(\mathrm{T}_{\mathrm{y}}\right)$

After the design of NF has been completed and the robustness should be tested, the optimal values of the ANFIS model have been used to implement and design the FPGA boards (four FPGA boards have been designed, one for each NF controller). Figure 12 shows the connection of the FPGA board with process system. First, a VHDL codes are downloaded into the FPGA chip (XILINX Spartan XC3S700AN) by using USB cable. Then, the Hirose 100-pin FX2 Edge connector (one port of the FPGA board) is used to connect the board (NF controller) with the suspension system. The error between the reference input and the system output has been applied as input to the A /D converter. The digital output of the A /D converter has been applied as input data to the FPGA boards. The FPGA board generates digital inputs to the NF controller (error and error rate). The NF controller generates a suitable digital control signal based on the rules that store in the FPGA chip. The digital control signal will be sent to the D/A convertor to generate an analog control signal that will be applied as input to the suspension system

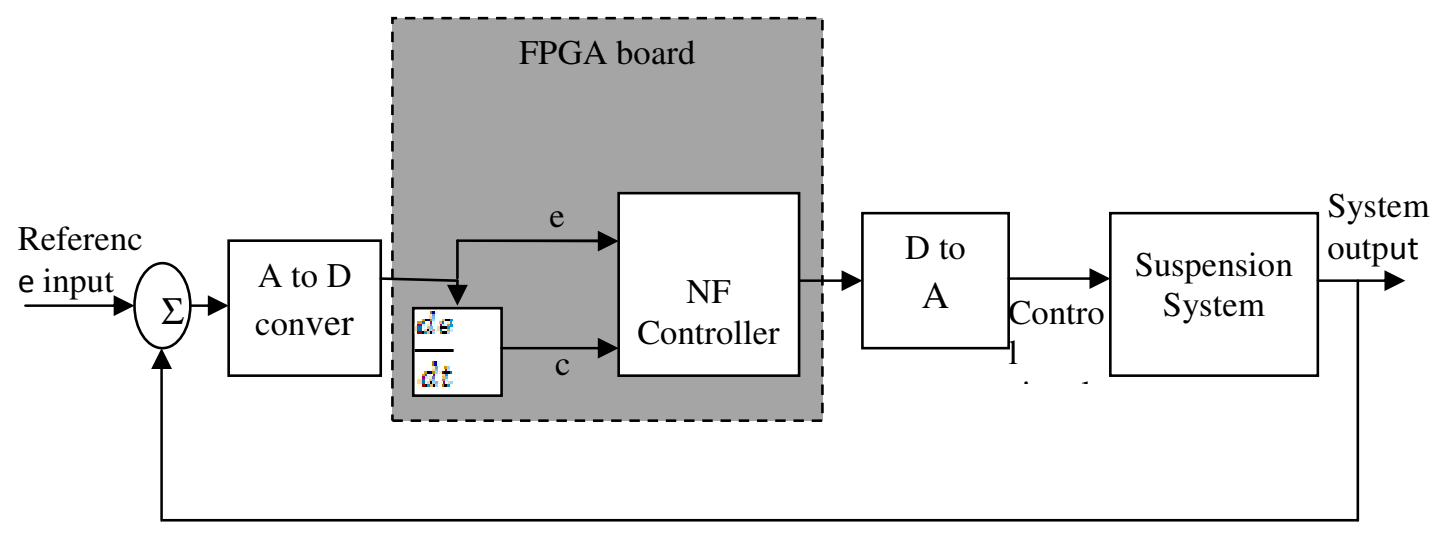

Figure 12. Layout of FPGA board with 
A test is performed to make sure that the NF controller used inside the FPGA-based design functions properly. The ModelSim XE III 6.4b program has been used to simulate the outputs of FPGA boards before using them as control systems for the suspension unit. The control signals, which have obtained form the simulation program (ModelSim XE III 6.4b), have been compared with the control signals which have obtained from Simulink design using Matlab Program. Figures 13-16 show the control signals obtained from each design (Simulation design and hardware design) for each controller
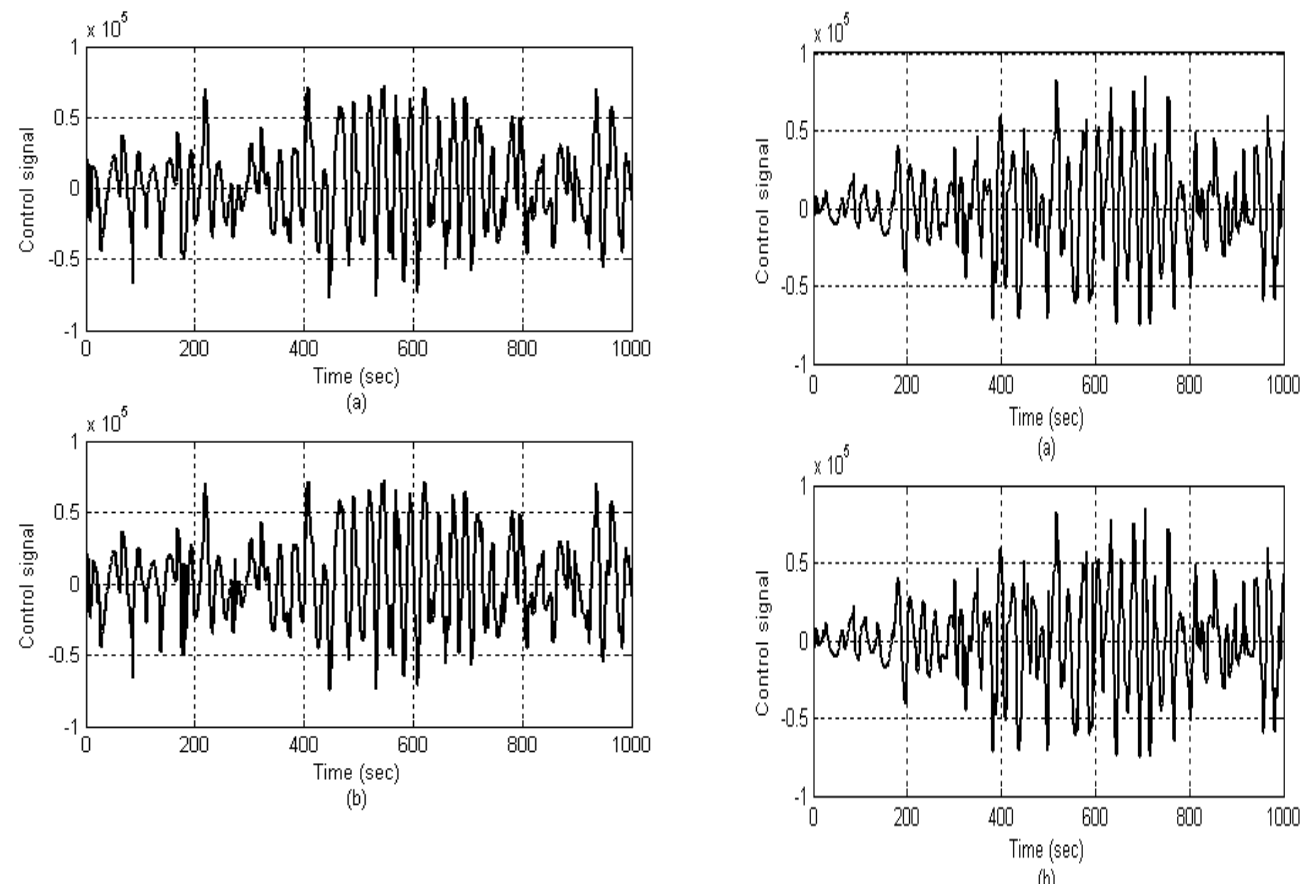

Figure 13. The control signal for first controller of (a).Simulation design. (b) Hardware design

Figure 14. The control signal for second controller of (a).Simulation design. (b) Hardware design 

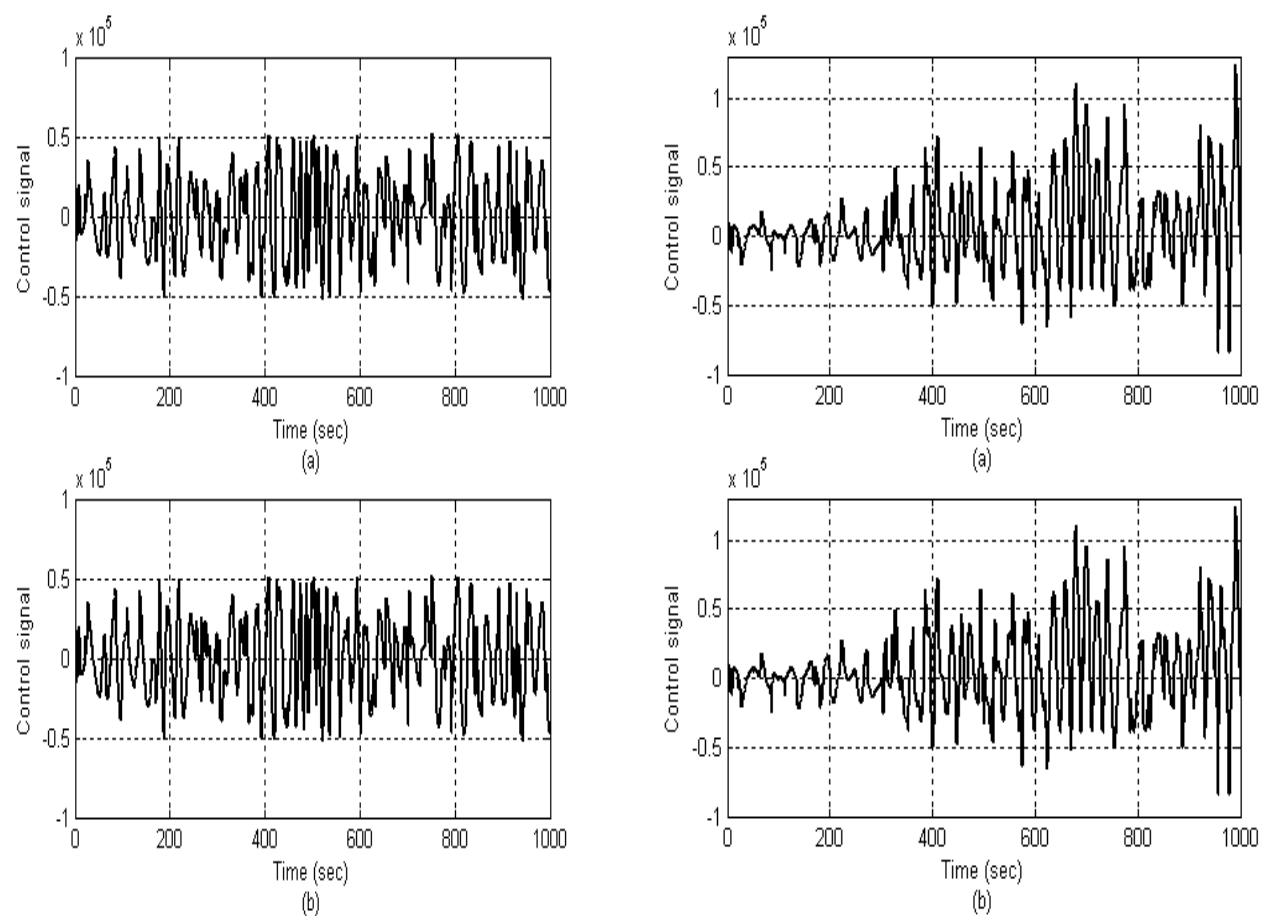

Figure 15. The control signal for control three of (a).Simulation design. (b).Hardware design

Figure 16. The control signal for control three of (a).Simulation design. (b) .Hardware design

\section{Conclusions}

The main objectives of designed the controller for a vehicle suspension system are to reduce the discomfort felt by passengers which arises from road roughness and to increase the ride handling associated with the pitching and rolling movements. In this paper, an ANFIS model has used to design the control system for full vehicle nonlinear active suspension system. The results have been compared with the performances of the FOPID controller and the NF controller in the case sudden disentrances occur. The proposed controller improves the vertical displacements at each corner of the vehicle to reach low level. It means that the ride comfort and the road handling have been improved. It has been confirmed that the proposed controller is more robust and more effective than the Fractional Order $\mathrm{PI}^{\lambda} \mathrm{D}^{\mu}$ (FOPID) controller. It provides a novel technique to be used to design NF controller for full vehicle nonlinear active suspension systems with hydraulic actuators. The optimal parameters of the NF controller have been used to design the controller hardware using the FPGA board. The results show that the control signals obtained from the FPGA boards are identical to the control signals which are obtained from design simulation. The results are encouraging and have proved the effectiveness both algorithm and hardware architecture. Therefore, the FPGA boards are effective to be used to control the full vehicle nonlinear active suspension systems with hydraulic actuators. 


\section{REFERENCES}

[1] Gilbert, B., A monolithic 16-channel Analog arry Normalizer. IEEE Journal of solid state circuits, 1984. 19(6): p. 956-963.

[2] Ishizuka, O., et al., Design of a Fuzzy Controller With Normalization Circuits. IEEE International Conference on Digital Objective Identifier, 1992: p. 1303-1308.

[3] Yamakawa, T., A Fuzzy Programmable Logic Array (Fuzzy PLA). IEEE International Conference on Digital Objective Identifier, 1992: p. 459-465.

[4] Manzoul, M.A. and D. Jayabharathi, FUZZY CONTROLLER ON FPGA CHIP. IEEE International Conference on Digital Objective Identifier, 1992: p. 1309-1316.

[5] Obaid, Z.A., et al., Analysis and Performance Evaluation of PD-like Fuzzy Logic Controller Design Based on Matlab and FPGA International Journal of Computer Science, 2010. 37(2).

[6] Economakos, G. and C. Economakos, A Run-Time Recongurable Fuzzy PID Controller Based on Modern FPGA Devices. Mediterranean Conference on Control and Automation, 2007: p. 1-6.

[7] Tipsuwanpornm, V., et al., Fuzzy Logic PID controller based on FPGA for process control. IEEE International Syposium on Industrial Electronics, 2004. 2: p. 1495-1500.

[8] Hu, B.S. and J. Li, The Fuzzy PID Gain Conditioner: Algorithm, Architecture and FPGA Implementation. IEEE International Conference on Industrial Technology, 1996: p. 621-624.

[9] Singh, B., et al., Design and VLSI implementation of Fuzzy Logic Controller International Journal of Computer and Network Security, 2009. 1(3).

[10] S.Poorani, et al., FPGA BASED FUZZY LOGIC CONTROLLER FOR ELECTRIC VEHICLE. Journal of The Institution of Engineers, 2005. 45(5): p. 1- 14.

[11] Cirstea, M., J. Khor, and M. McCormick, FPGA Fuzzy Logic Controller for Variable Speed Generator. IEEE International Conference on Control Application, 2001: p. 5-7.

[12 Barriga, A., et al., Modelling and implementation of fuzzy systems based on VHDL. International Journal of Approximate Reasoning, 2006. 41: p. 164-178.

[13] Blake, J.J., et al., The implementation of fuzzy systems, neural networks and fuzzy neural networks using FPGAs Information Science, 1998. 112: p. 151-168.

[14] Ando, Y. and M. Suzuki, Control of Active Suspension Systems Using the Singular Perturbation method. Control Engineering Practice, 1996. 4(33): p. 287-293.

[15] Merritt, H., Hydraulic Control Systems. 1969, USA: John wiley and Sons,Inc.

[16] xue, D., Y. Chen, and D. Atherton, Linear Feedback Controller Analysis and Design with MATLABE. 2007, USA: The Society for Industrial and Applied Mathematics.

[17] Back, T., Evolutionary Algorithms in Theory and Practice. 1996, London, UK: Oxford University Press.

[18] Nguyen, H.T., et al., A First Course in Fuzzy and Neural Control. 2003, USA: Chapman \& Hall/ CRC.

[19] Jang, J.-S.R., ANFIS: Adaptive Network Based Fuzzy Inference System. IEEE Transaction on System, Man and Cybernetics 1993. 23(3): p. 665-686. 


\section{Authors}

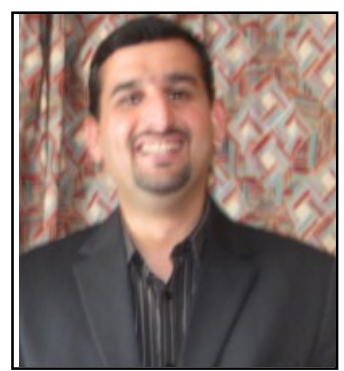

Ammar Aldair was born in Basrah, Iraq. He received his B.Sc. Degree in Electrical Engineering from University of Basrah, Iraq in 2000. In 2003, he received his M.Sc. Degree in Control and Systems Engineering from University of Basrah, Iraq. From 2003-2008, he was a Lecturer in Electrical Department, University of Basrah, Iraq. He taught many subjects such as: Mathematics, Logic Systems, Electrical Circuits, Electronic Circuits, Control Systems and Advance Control systems. In 2008, he had a scholarship from Iraqi government to get the DPhil Degree in Intelligent Control Systems from UK. Currently, he is a DPhil student in School of Engineering and Design, University of Sussex, UK. His current research interests in Intelligent Control Systems.

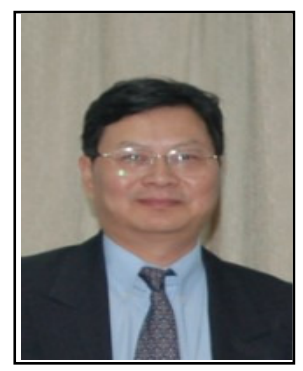

Weiji Wang was born in China. He received his DPhil degree from Oxford University in 1993. Currently he is a senior lecturer in School of Engineering and Design, University of Sussex. His current research interests in Automotive Dynamics and Control. 